\title{
Influência da diabetes e a prática de exercício físico e atividades cognitivas e recreativas sobre a função cognitiva e emotividade em grupos de terceira idade
}

\author{
Influence of diabetes and physical exercise, mental and recreational activities practice on the \\ cognitive function and emotionality in aging groups
}

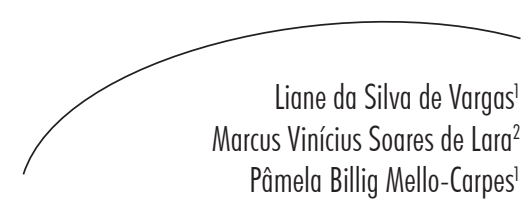

Resumo

Objetivo: Neste estudo buscou-se verificar a influência da participação em atividades de grupos de terceira idade (GTI), envolvendo a prática de exercícios físicos e atividades cognitivas e recreativas, sobre a função cognitiva e aspectos emocionais, como ansiedade e depressão, de idosos diabéticos e não diabéticos. Métodos: Participaram deste estudo descritivo transversal 158 idosos, subdivididos em quatro grupos: (1) idosos não participantes de GTI e não diabéticos; (2) idosos não participantes de GTI e diabéticos; (3) idosos participantes de GTI e não diabéticos; e (4) idosos participantes de GTI e diabéticos. Todos os idosos foram submetidos a um protocolo de avaliação cognitiva (Miniexame do Estado Mental-MEEM, Teste de Recordação Imediata e Tardia Livre de Palavras - RIP e RTP, respectivamente, e Teste de Reconhecimento de Faces Famosas-FF), ansiedade (IDATE) e depressão (Escala de Depressão GeriátricaEGD). Para comparação entre os grupos, utilizou-se ANOVA de uma via para os dados paramétricos e teste de Kruskal-Wallis para os não paramétricos. Resultados: Os idosos participantes do estudo tinham idade média de 72,63 $\pm 6,84$ anos (75\% mulheres e $25 \%$ homens). Os resultados demonstraram que os idosos do grupo 2 (diabéticos e não participantes de GTI) apresentaram menores escores cognitivos do que os do grupo 3 (não diabéticos e participantes de GTI) ( $\mathrm{p}=0,012$ no MEEM; $\mathrm{p}=0,028$ na RIP; $\mathrm{p}=0,011$ na EGD). Conclusão: Pode-se afirmar que a associação da diabetes mellitus com um estilo de vida menos ativo, sem a prática de exercícios físicos e atividades recreativas e cognitivas, possivelmente representa um fator de risco para a aceleração das perdas cognitivas que acompanham o processo de envelhecimento.

\footnotetext{
Programa de Pós-graduação em Bioquímica. Universidade Federal do Pampa. Uruguaiana, RS, Brasil.

2 Curso de Fisioterapia, Centro de Ciências da Saúde. Universidade Federal do Pampa. Uruguaiana, RS, Brasil.
}

Palavras-chave: Diabetes Mellitus Tipo 2. Cognição. Transtorno Cognitivo. Atividade Física. Atividade Cognitiva. 


\section{Abstract}

Objective: This study aimed to determine the influence of participation in third age groups (TAG), involving the practice of physical exercise and cognitive and recreational activities, on cognitive function and emotional issues such as anxiety and depression in diabetic and non-diabetic elderly patients. Methods: In this cross sectional study, participated 158 older adults divided into four groups: (1) elderly non-participants in TAG and non-diabetic; (2) elderly non-participants in TAG and diabetic; (3) elderly participants in TAG and non-diabetic; and (4) elderly participants in TAG and diabetic. All of them were submitted to a protocol of cognitive assessment (Mini-Mental State-MMSE, Immediate and Delayed Recall Test Free Words - RIP and RTP, and Recognition of Famous Faces Test-FF), anxiety (STAI) and depression (Geriatric Depression Scale-EGD). For comparison between groups, we used one-way ANOVA for parametric data and the Kruskal-Wallis test for non-parametric data. Results: The mean age of the sample was $72.63 \pm 6.84$ years old ( $75 \%$ women and $25 \%$ men). The results demonstrate that the elderly group 2 (diabetic and non-participants EG) have lower cognitive scores than group 3 (non-diabetic participants and EG) ( $p=0.012$ in MMSE, $\mathrm{p}=0.028$ in the IWR, $\mathrm{p}=0.011$ in GDS). Conclusion: It can be argued that the association between diabetes mellitus and less active lifestyle, without the practice of physical exercise and recreational and cognitive activities, possibly represents a risk factor for the acceleration of cognitive losses that follow the aging process.
Key words: Diabetes Mellitus, Type 2. Cognition. Cognition Disorders. Physical Activity. Cognitive Activity.

\section{INTRODUÇÃo}

Nos últimos anos, o perfil demográfico e epidemiológico da população brasileira tem mudado rapidamente. Ao contrário da transição demográfica, que ocorreu de forma lenta e acompanhando a elevação da qualidade de vida nos países desenvolvidos, no Brasil essa mudança vem acontecendo de forma rápida e desvinculada de uma política social favorável, sem melhora simultânea da qualidade de vida desses indivíduos. ${ }^{1}$ Segundo Veras, ${ }^{2}$ anualmente 650 mil novos idosos são incorporados à população, e a maioria apresenta doenças crônicas não transmissíveis e limitações funcionais.

A expectativa de vida vem aumentando linearmente, assim como as doenças crônicas e desabilidades, ${ }^{3}$ as quais têm influência direta na autonomia e qualidade de vida da população idosa. Diante desse contexto, a busca por um envelhecimento saudável e por uma boa qualidade de vida na terceira idade é fundamental.

Sabe-se que o processo de envelhecimento envolve, entre outras coisas, um declínio cognitivo fisiológico, que se dá em detrimento do processo de degeneração do sistema nervoso, o qual inclui diversas alterações estruturais. Um exemplo é a redução do volume observado nas regiões do córtex pré-frontal e do lobo temporal medial, ambas críticas para a memória episódica., ${ }^{4,5}$

É preciso considerar, porém, que existem muitas variações no desenvolvimento do esquecimento fisiológico decorrente do envelhecimento. Segundo Izquierdo, ${ }^{6}$ a perda neuronal gradual parece ocorrer mais rapidamente em indivíduos que não costumam praticar seu intelecto e suas funções cognitivas do que em indivíduos intelectualmente ativos; outro fator que pode agravar o declínio fisiológico é quando este é acompanhado pela presença de determinadas condições patológicas.

Dentre as principais doenças crônicas que acometem a população, o diabetes mellitus (DM) tem sido considerado um grave problema de saúde pública, uma vez que a prevalência da doença aumenta com a idade. ${ }^{7}$ Trata-se de uma desordem metabólica crônica caracterizada pelo aumento anormal do nível de glicose no 
sangue (hiperglicemia). No Brasil existem cerca de 500 mil diabéticos, 450 mil fazendo uso da insulina e quase dois milhões desconhecendo ter diabetes. ${ }^{8}$ Além disso, estima-se que em 2030 o número de pessoas acometidas pela DM dobrará, devido ao processo de urbanização, obesidade e envelhecimento.?

A DM apresenta-se em duas principais formas: DM tipo 1 (DM1) e DM tipo 2 (DM2). Esta classificação sebaseianaetiologiadahiperglicemia, bem como na resposta do indivíduo à insulina. A DM1 corresponde a aproximadamente $5-10 \%$ dos casos de DM e é caracterizada pela deficiência de insulina, enquanto que a DM2 caracteriza-se pela incapacidade da insulina em exercer adequadamente seus efeitos nos tecidosalvos, condição que cronicamente está associada a disfunção e falência de vários órgãos, o que pode interferir negativamente nas tarefas de vida diária desses indivíduos. A DM2 corresponde a 90-95\% dos casos diagnosticados. ${ }^{10,11}$

De acordo com Ozturk, ${ }^{12}$ idosos que apresentam algum tipo de síndrome metabólica comumente terão as dimensões físicas e emocionais da vida afetadas, com limitação das atividades diárias, independência e qualidade da vida. No entanto, o declínio cognitivo pode ser retardado quando associado a hábitos de vida específicos. Ainda, estudos recentes têm demonstrado estreita relação entre a DM e a doença de Alzheimer (DA), uma vez que idosos diabéticos apresentam prejuízo em seu desempenho cognitivo quando comparados a indivíduos não diabéticos, assim como os diabéticos têm risco aumentado de desenvolver demências, em especial a DA. ${ }^{13,14}$

Existem alternativas que, acredita-se, podem prevenir ou manter as funções cognitivas e a qualidade de vida do idoso, dentre as quais se destaca a prática de exercícios físicos e atividades cognitivas e/ou recreativas, realizadas sobretudo em grupos específicos para idosos (grupos de terceira idade - GTI). Exercício físico, além de auxiliarem no tratamento e controle da DM, têm sido reportados como auxiliares, mantendo ou melhorando funções cognitivas, como a memória, em diversas situações. ${ }^{15,16}$ Estudos demonstram que a prática de exercícios físicos atenua o declínio cognitivo associado ao envelhecimento em humanos; além disso, os indivíduos que são fisicamente mais ativos acumulam benefícios de proteção contra demências, especialmente a DA. ${ }^{16,17}$

Sabe-se também que a diminuição da atividade cognitiva contribui, e muito, para que o declínio cognitivo aconteça de maneira mais avançada. Estudos têm demonstrado o benefício da prática de atividades cognitivas e/ou recreativas no desempenho cognitivo de idosos, uma vez que a participação em atividades deste cunho contribui para o aumento da atividade neuronal, cuja rede de transmissão é responsável pela plasticidade e dinâmica cerebral. ${ }^{18}$ Assim, idosos submetidos à prática de atividades cognitivas apresentam melhora em seu desempenho cognitivo, bem como ampliação da rede social e o sentimento de autoeficácia. ${ }^{19}$

Diante do exposto, este estudo procurou verificar a influência da participação em grupos de terceira idade (GTI), envolvendo a prática de exercícios físicos e atividades cognitivas e recreativas, sobre a função cognitiva e aspectos emocionais, como ansiedade e depressão, de idosos diabéticos e não diabéticos.

\section{METODOLOGIA}

Local e sujeitos do estudo

Este estudo descritivo transversal foi desenvolvido em um Centro de Convivência de idosos, em grupos de idosos (entidades que reúnem idosos para prática de exercícios físicos e atividades cognitivas e recreativas em grupo), e em duas Unidades Básicas de Saúde (UBS) do município de Uruguaiana-RS. Participaram 158 idosos com idade acima de 60 anos, selecionados por conveniência. A coleta dos dados ocorreu entre os meses de março e novembro de 2012, totalizando um período de oito meses.

Os idosos foram subdivididos em quatro grupos, de acordo com suas características: grupo 1 - composto por 40 idosos, sendo 32 mulheres e 
oito homens, todos não participantes de GTI e não diabéticos; grupo 2 - composto por 40 idosos, sendo 28 mulheres e 12 homens, todos não participantes de GTI e diabéticos; grupo 3 - composto por 40 idosos, sendo 34 mulheres e seis homens, todos participantes de GTI e não diabéticos; e grupo 4 - composto por 38 idosos, sendo 25 mulheres e 13 homens, todos participantes de GTI e diabéticos.
Não foram incluídos na análise dos dados os idosos que apresentassem diagnóstico de DM tipo I ou de DM tipo II há menos de três meses e/ou idosos que participassem de GTI a um período inferior a três meses. Todos os indivíduos foram submetidos a um mesmo protocolo de avaliação, o qual foi aplicado por pesquisadores previamente treinados (figura 1).

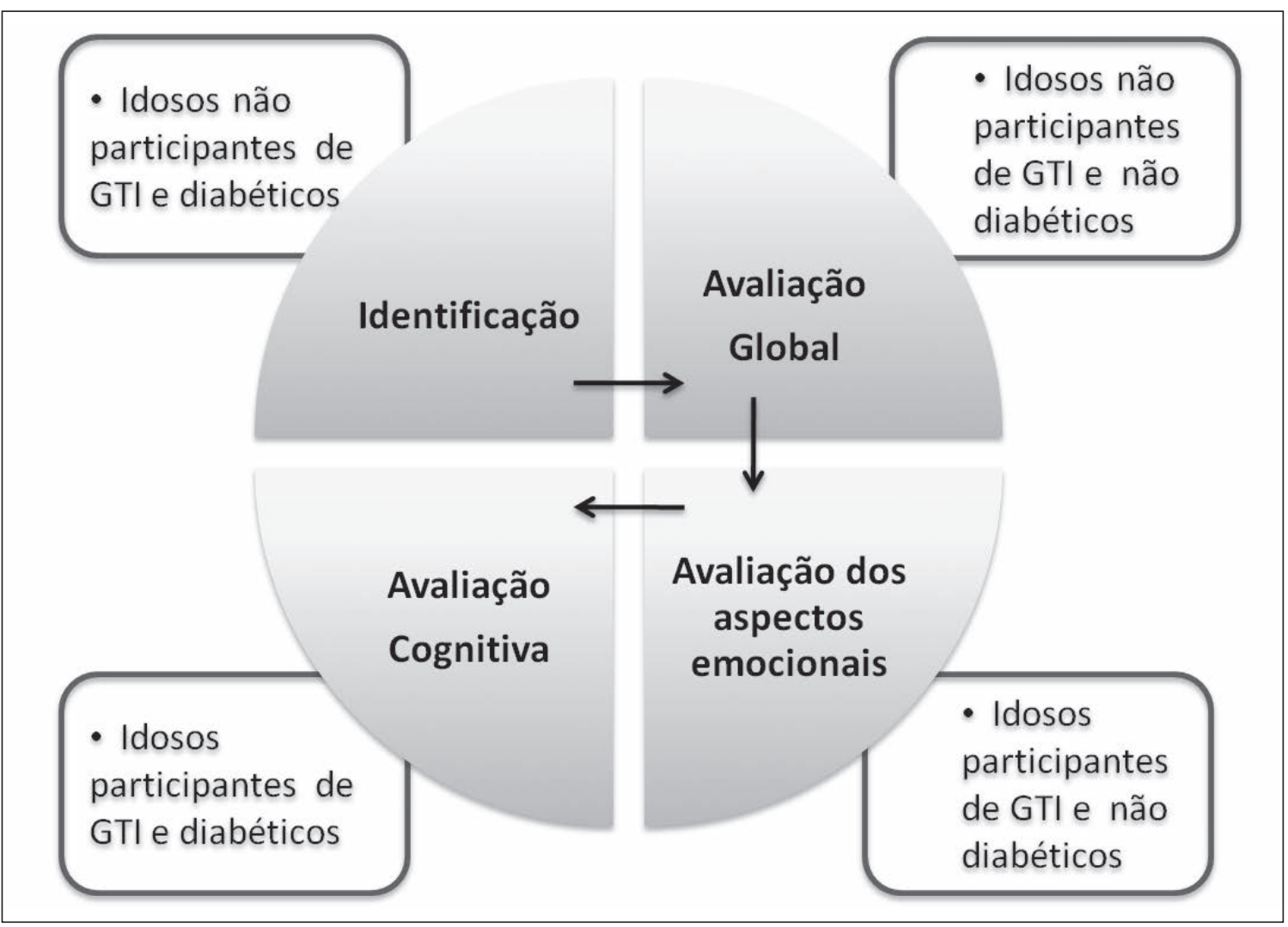

GTI= grupos de terceira idade.

Figura 1. Subgrupos de idosos e etapas do protocolo de avaliação. Uruguaiana-RS, 2012.

Procedimentos

O protocolo de avaliação dos idosos consistiu em três etapas consecutivas: (1) avaliação global; (2) avaliação cognitiva; (3) avaliação dos aspectos emocionais.

\section{(1) Avaliação global}

Nesta etapa do protocolo, foram investigados os hábitos de vida e nível de saúde de cada idoso por meio de um formulário com questões abertas incluindo perguntas relacionadas a idade, 
realização ou não de exercícios físicos e atividades cognitivas, participação ou não em GTI (e período de participação, se for o caso) e presença de doenças crônicas (e tempo da doença, se for o caso). Esta primeira etapa auxiliou na adequação de cada idoso avaliado em um dos quatro grupos de idosos do estudo.

\section{(2) Avaliação cognitiva}

Nesta etapa, os idosos foram submetidos a uma avaliação da função cognitiva por meio dos seguintes instrumentos:

2.1 Miniexame do Estado Mental (MEEM): instrumento que permite a avaliação geral da função cognitiva. É dividido em cinco seções que fornecem informações sobre diferentes aspectos cognitivos: orientação, memória imediata, atenção e cálculo, evocação e linguagem. ${ }^{20}$ Este instrumento permite o escore imediato mediante a soma dos pontos atribuídos a cada tarefa realizada com sucesso, o qual varia de 0-30 pontos. Neste estudo utilizou-se o ponto de corte limite de 19/20 pontos para idosos sem educação formal e 23/24 para idosos com história escolar prévia. ${ }^{21}$

2.2 Teste de Recordação Imediata e Tardia de Palavras (RIP-RTP): teste utilizado para avaliar a memória declarativa, fornecendo indícios de memória de curto prazo e de longo prazo, além de memória auditiva. ${ }^{22}$ Para realização deste teste, o idoso foi colocado em uma sala reservada, sendo orientado a prestar atenção na lista de palavras que o pesquisador iria ler. $\mathrm{O}$ idoso foi orientado a memorizar o máximo de palavras possíveis e a ordem em que as mesmas foram citadas. Posteriormente, o pesquisador leu, de maneira clara e pausada, dez palavras. Imediatamente após, e dez minutos após a leitura, o idoso era solicitado a evocar as palavras que o mesmo recordava. $\mathrm{O}$ escore deste teste varia de 0-10 pontos para cada domínio, recordação imediata e recordação tardia. Quanto maior a pontuação, melhor o desempenho do idoso neste teste.

2.3 Teste de Reconhecimento de Faces Famosas (FF): teste que permite avaliar a aptidão para reconhecer faces de pessoas famosas, imediatamente após sua exposição. É indicado para avaliar as funções da memória e a percepção visual. ${ }^{23}$ Para este teste, foram utilizadas dez imagens de atores, apresentadores, esportistas e/ ou políticos famosos e amplamente conhecidos. Os idosos deveriam identificálos imediatamente, ou em, no máximo, um minuto após a apresentação. $\mathrm{O}$ escore deste teste varia de 0-20 pontos; em cada figura apresentada o sujeito recebe 1 ponto, no caso da identificação da profissão da pessoa famosa, ou 2 pontos, quando a identificação for completa (profissão da pessoa, bem como nome da mesma). Dessa forma, quanto maior a pontuação neste teste, melhor $\mathrm{o}$ desempenho do idoso.

\section{(3) Avaliação dos aspectos emocionais}

A última etapa visou avaliar os aspectos emocionais do idoso, mediante os seguintes instrumentos:

3.1 Escala Geriátrica de Depressão (EGD): instrumento utilizado para a detecção de indícios de depressão em pessoas idosas, oferecendo mensurações válidas e confiáveis para o rastreio de transtornos depressivos. É composto de 30 perguntas com duas possibilidades de resposta, "sim" e "não", e conforme o escore final, o idoso é classificado como "com suspeita de depressão" ou "sem suspeita de depressão". ${ }^{24}$ 
3.2 Inventário de Ansiedade Traço-Estado (IDATE): questionário de autoavaliação dividido em duas partes. A primeira avalia a ansiedade-traço e a segunda avalia a ansiedade-estado, sendo cada uma destas partes compostas de 20 afirmações. Ao responder ao questionário, o indivíduo deve levar em consideração uma escala de quatro itens que variam de 1 a 4 , sendo que "estado" significa como o sujeito se sente "no momento" e o "traço" como ele "geralmente se sente". O escore de cada parte varia de 20 a 80 pontos, sendo que, quanto mais baixo os escores, menor o grau de ansiedade. ${ }^{25}$

\section{Análise dos resultados}

A normalidade dos dados foi verificada por meio do teste de Shapiro-Wilk. Para comparação dos resultados dos diferentes grupos, utilizou-se ANOVA de uma via seguida de post hoc de Tukey para os dados paramétricos. Para os dados não paramétricos, utilizou-se o teste de KruskalWallis, seguido de post hoc de Dunn ou teste de Mann-Whitney, no caso de comparação entre dois grupos. Foram considerados significativos resultados com valor de $\mathrm{p} \leq 0,05$.

\section{Aspectos éticos}

Todos os princípios éticos contidos na Declaração de Helsinki e na legislação pertinente foram seguidos com rigor na realização deste estudo, que foi aprovado pelo Comitê de Ética em Pesquisa da Universidade Federal do Pampa - Unipampa (registro no 001/2011). Além disso, todos os participantes deste estudo assinaram o Termo de Consentimento Livre e Esclarecido (TCLE) para a participação na pesquisa.

\section{RESULTADOS}

A amostra foi composta por 158 idosos com média de idade de 72,61 $\pm 6,83$ anos, sendo 120 mulheres $(75,94 \%)$ e 38 homens $(24,05 \%)$. Os idosos foram divididos entre os quatro grupos do estudo, de acordo com os critérios previamente explicitados. As características de cada subgrupo que compôs a amostra do estudo estão descritas na tabela 1.

Tabela 1. Características da amostra. Uruguaiana-RS, 2012.

\begin{tabular}{lccccc}
\hline Variáveis & $\begin{array}{c}\text { Não } \\
\text { participantes } \\
\text { de GTI e não } \\
\text { diabéticos } \\
(\mathrm{n}=40)\end{array}$ & $\begin{array}{c}\text { Não } \\
\text { participantes } \\
\text { de GTI e } \\
\text { diabéticos } \\
(\mathrm{n}=40)\end{array}$ & $\begin{array}{c}\text { Participantes } \\
\text { de GTI e não } \\
\text { diabéticos } \\
(\mathrm{n}=40)\end{array}$ & $\begin{array}{c}\text { Participantes } \\
\text { de GTI e } \\
\text { diabéticos } \\
(\mathrm{n}=38)\end{array}$ & $\begin{array}{c}\text { Valor } \\
\text { de p }\end{array}$ \\
\hline Idade (anos) & $72,68 \pm 7,12$ & $69,11 \pm 5,27$ & $74,85 \pm 7,00^{@}$ & $73,81 \pm 6,50^{@}$ & $0,004^{*}$ \\
Gênero (\%/n) & $80 \%(32) \mathrm{F}$ & $70 \%(28) \mathrm{F}$ & $85 \%(34) \mathrm{F}$ & $68 \%(25) \mathrm{F}$ & - \\
& $20 \%(8) \mathrm{M}$ & $30 \%(12) \mathrm{M}$ & $15 \%(6) \mathrm{M}$ & $32 \%(13) \mathrm{M}$ & \\
Tempo de DM (anos) & - & $8,38 \pm 5,24$ & - & $10,06 \pm 10,83$ & 0,795 \\
Tempo de prática de & - & - & $10,4 \pm 16,86$ & $17,43 \pm 21,51$ & 0,078 \\
exercício físico (anos) & & & & & \\
\hline
\end{tabular}

$\mathrm{DM}=$ diabetes mellitus; ${ }^{\mathrm{p}} \mathrm{p} \leq 0,05$ na ANOVA de uma via; ${ }^{\circledR}$ diferente do grupo não participante de GTI e diabético no post hoc de Tukey. 
Dentre as características da amostra, em relação à idade, verificou-se que os idosos participantes de GTI apresentaram idade superior em relação ao grupo diabético não participante de GTI ( $\mathrm{p}=0,04$, ANOVA de uma via seguida de post hoc de Tukey). Não houve diferença entre o tempo de prática de exercício físico e entre os grupos praticantes e no tempo de diagnóstico de DM nos grupos diabéticos (teste de MannWhitney).

Os resultados obtidos durante a aplicação das etapas 2 e 3 do protocolo de avaliação estão descritos na tabela 2 .

Tabela 2. Resultados obtidos nas etapas 2 e 3 do protocolo de avaliação. Uruguaiana-RS, 2012.

\begin{tabular}{|c|c|c|c|c|c|}
\hline Variáveis & $\begin{array}{c}\text { Não } \\
\text { participantes } \\
\text { de GTI e não } \\
\text { diabéticos } \\
(\mathrm{n}=40)\end{array}$ & $\begin{array}{c}\text { Não } \\
\text { participantes } \\
\text { de GTI e } \\
\text { diabéticos } \\
(\mathrm{n}=40)\end{array}$ & $\begin{array}{l}\text { Participantes } \\
\text { de GTI e não } \\
\text { diabéticos } \\
(\mathrm{n}=40)\end{array}$ & $\begin{array}{l}\text { Participantes } \\
\text { de GTI e } \\
\text { diabéticos } \\
(\mathrm{n}=38)\end{array}$ & $\begin{array}{l}\text { Valor } \\
\text { de } \mathrm{p}\end{array}$ \\
\hline MEEM & $22,55 \pm 3,13$ & $21,98 \pm 3,48^{\#}$ & $24,28 \pm 2,70$ & $22,71 \pm 3,11$ & $0,009^{*}$ \\
\hline MEEM - orientação & $8,58 \pm 1,17$ & $8,60 \pm 1,34$ & $8,90 \pm 1,06$ & $8,61 \pm 1,46$ & 0,731 \\
\hline MEEM - recordação & $2,97 \pm 0,18$ & $2,97 \pm 0,17$ & $3,00 \pm 0,00$ & $2,97 \pm 0,16$ & 0,798 \\
\hline MEEM - atenção e cálculo & $1,32 \pm 1,14$ & $1,28 \pm 1,11^{\&}$ & $2,16 \pm 1,65$ & $1,64 \pm 1,54$ & $0,034^{@}$ \\
\hline MEEM - evocação & $1,64 \pm 1,25$ & $1,48 \pm 1,34^{\&}$ & $2,20 \pm 0,96$ & $2,06 \pm 1,09$ & $0,036^{@}$ \\
\hline MEEM - linguagem & $7,74 \pm 0,96$ & $7,70 \pm 0,81$ & $7,67 \pm 0,94$ & $7,46 \pm 1,06$ & 0,784 \\
\hline $\begin{array}{l}\text { Recordação Livre de } \\
\text { Palavras - Imediata }\end{array}$ & $4,65 \pm 1,14$ & $3,43 \pm 4,16^{\&}$ & $5,05 \pm 1,41$ & $4,13 \pm 5,12$ & $0,028^{\circledR}$ \\
\hline $\begin{array}{l}\text { Recordação Livre de } \\
\text { Palavras - Tardia }\end{array}$ & $2,90 \pm 1,60$ & $2,37 \pm 1,67$ & $3,53 \pm 2,13$ & $2,95 \pm 2,12$ & 0,069 \\
\hline Faces Famosas & $16,53 \pm 3,52$ & $15,78 \pm 3,19$ & $17,03 \pm 3,48$ & $16,55 \pm 3,16$ & 0,417 \\
\hline $\begin{array}{l}\text { Escala Geriátrica de } \\
\text { Depressão }\end{array}$ & $8,30 \pm 5,25^{\&}$ & $8,60 \pm 3,57^{\&}$ & $5,94 \pm 3,20$ & $5,97 \pm 8,30$ & $0,011^{@}$ \\
\hline IDATE - Traço & $47,15 \pm 9,71$ & $49,13 \pm 6,37$ & $49,51 \pm 7,56$ & $46,74 \pm 5,64$ & 0,263 \\
\hline IDATE - Estado & $45,43 \pm 9,35$ & $46,00 \pm 9,65$ & $46,44 \pm 4,65$ & $47,82 \pm 6,99$ & 0,446 \\
\hline
\end{tabular}

*p $\leq 0,05$ na ANOVA de uma via; \# diferente dos idosos participantes de GTI e não diabéticos no post hoc de Tukey; @ p $\leq 0,05$ no teste de Kruskall-Wallis; \& diferente dos idosos participantes de GTI e não diabéticos no post hoc de Dunn. 
Com relação à avaliação cognitiva, observouse que no escore total do MEEM o grupo diabético e não participante de GTI apresentou pior desempenho cognitivo, quando comparado ao grupo de idosos participantes de GTI e não diabéticos $(\mathrm{p}=0,009$, ANOVA de uma via seguida de post hoc de Tukey). A diferença entre esses dois grupos também foi observada em relação às esferas cognitivas avaliadas separadamente por meio do MEEM, sendo que o grupo diabético e não participante de GTI teve pior desempenho de atenção e cálculo ( $\mathrm{p}=0,034$, teste de Kruskall Wallis seguido de post hoc de Dunn) e evocação ( $\mathrm{p}=0,036$; teste de Kruskall Wallis seguido de post hoc de Dunn). Nas demais esferas cognitivas avaliadas pelo MEEM (orientação, recordação e linguagem), não foram observadas diferenças entre os grupos.

No teste de RIP, também foi observada diferença significativa entre os mesmos grupos, ratificando que o grupo de idosos diabéticos não participantes de GTI apresentou capacidade de recordação imediata inferior quando comparado ao grupo de idosos não diabéticos e participantes de GTI ( $p=0,028$, teste de Kruskall Wallis seguido de post hoc de Dunn). Essa diferença, no entanto, não foi observada no teste de recordação tardia (RTP) e no teste de faces famosas. Além disso, é válido ressaltar que os piores escores cognitivos, encontrados no grupo de idosos diabéticos e não praticantes de GTI, contemplam o grupo com menor média de idade (69,11 $\pm 5,27$ anos).

Em relação aos aspectos emocionais, verificou-se que há tendência ao desenvolvimento de depressão nos idosos do grupo não diabéticos e não participantes de GTI, bem como do grupo dos diabéticos e não participantes de GTI, quando comparados ao grupo de participantes de GTI não diabéticos ( $\mathrm{p}=0,011$ no teste de Kruskall-Wallis seguido de post-hoc de Dunn). Nas avaliações de ansiedade (IDATE), não foram observadas diferenças entre os grupos.

\section{DISCUSSÃO}

Os resultados deste estudo permitiram verificar que idosos diabéticos e não participantes de GTI apresentaram desempenho cognitivo significativamente pior do que idosos participantes de GTI e não diabéticos, indicando que provavelmente a associação da DM com uma possível privação da prática de exercícios físicos e atividades cognitivas e/ou recreativas pode prejudicar a função cognitiva de idosos. Cabe destacar que se supõe que a associação da DM com a inatividade (física, cognitiva e recreativa) é o fator contribuinte nesta questão, porque nos demais grupos, nos quais somente um fator estava presente (ou a DM, ou a não participação em GTI - inatividade), não se observou piora da função cognitiva.

Outros estudos, como o de Coelho et al., ${ }^{26}$ evidenciam que a presença de doenças crônicas e o baixo nível de atividade física são fatores contribuintes para a inabilidade funcional de indivíduos idosos. Além disso, a prática de exercício físico tem apresentado efeito protetor em relação à função cognitiva em idosos, evidenciando ser uma intervenção não farmacológica promissora para prevenir o declínio cognitivo. ${ }^{16}$ Não se pode deixar de considerar que os idosos participantes de GTI, por mais que não realizem exercícios físicos devidamente controlados em sua intensidade e frequência, apresentam-se mais ativos que os idosos que não frequentam esses grupos e não praticam outras atividades. Assim, este fato pode contribuir para explicar os resultados obtidos no presente estudo, que mostraram que a simples presença de uma doença crônica, no caso a $\mathrm{DM}$, por si só não causa prejuízo significativo para a função cognitiva, mas quando associada à inatividade física e social, pode causar.

A maioria dos GTIs também estimula e propõe a realização de atividades cognitivas e ocupacionais pelos idosos. Estudos que analisaram a repercussão da prática de atividades cognitivas no desempenho cognitivo de idosos demonstraram relação positiva entre essas variáveis. ${ }^{18,19}$ A participação de 46 idosos em uma "oficina de memória" relatada por Oliveira em 2012, ${ }^{19}$ por exemplo, promoveu melhora significativa no desempenho cognitivo da maioria dos indivíduos avaliados, evidenciando 
o benefício desta prática. Já foi demonstrado também que indivíduos que participam ativamente de atividades cognitivas, tais como jogos de xadrez, leituras e escrita, têm menor probabilidade de desenvolver algum tipo de demência. ${ }^{27,28}$

Outro aspecto relevante na participação em GTI é o convívio social que esses espaços proporcionam ao idoso. Em estudo prospectivo realizado com idosos, a fim de avaliar a relação entre atividades de lazer e o risco de desenvolver demências, foi observado que o envolvimento de idosos em atividades sociais está relacionado com um menor risco de os mesmos desenvolverem algum tipo de demência. ${ }^{29}$ Ainda de acordo com Hall e colaboradores, ${ }^{28}$ a prática de atividades cognitivas, individualmente ou em grupo, mesmo com início tardio, ou seja, durante a terceira idade, tem influência sobre a reserva cognitiva, independentemente do nível educacional do idoso. Em seu estudo, ${ }^{28}$ foi investigada a influência da participação autorrelatada em atividades de lazer cognitivamente estimulantes no declínio acelerado da memória em idosos no estágio pré-clinico de demência. Foram avaliados 101 idosos, integrantes do estudo de coorte Bronx Aging Study, por meio do teste de memória seletiva de Buschke. Cada dia de atividade cognitiva autorrelatada no início do estudo, incluindo escrita, leitura, palavras cruzadas, jogos de tabuleiro ou cartas, discussões em grupo e reprodução musical, atrasou o início do declínio acelerado da memória em 0,18 anos, o que reforça os achados deste estudo, uma vez que os idosos integrantes de GTI apresentaram melhor desempenho cognitivo do que idosos não participantes de GTI.

Os estudos mencionados corroboram os achados deste estudo, reforçando a ideia de que tanto o diabetes como a privação de práticas de exercícios físicos e atividades cognitivas e convívio social contribuem para o aceleramento do declínio cognitivo, com consequente repercussão na autonomia e qualidade de vida desses indivíduos. No entanto, quando associadas, sua repercussão sobre a cognição é ainda mais alarmante. Cabe ressaltar que o grupo de idosos diabéticos e não participantes de GTI apresentou os piores escores cognitivos, mesmo considerando que os idosos deste grupo apresentaram média de idade significativamente menor que outros grupos. Isso demonstrou que o prejuízo cognitivo encontrado neste grupo é consequência da associação das variáveis supracitadas, e não do processo fisiológico do envelhecimento em si.

Em relação ao aspecto emocional, verifica-se que existe maior tendência ao desenvolvimento de depressão nos idosos do grupo de diabéticos e não participantes de GTI e nos não diabéticos e não participantes de GTI, quando comparados ao grupo de participantes de GTI e não diabéticos. Recentes achados têm evidenciado associação entre sintomas de declínio cognitivo e depressão em indivíduos diabéticos. Em revisão sistemática realizada por Roy \& Lloyd, ${ }^{30}$ os autores afirmam que a DM é fator de risco para o desenvolvimento de depressão. Silva et al. ${ }^{31}$ evidenciaram uma possível relação entre a resistência à insulina no cérebro e também na periferia, com a demência e a depressão em indivíduos com DM2. Campbell et al. ${ }^{32}$ também demonstraram que baixos níveis de atividade física têm sido relacionados com insuficiente saúde mental, depressão e percepção de estresse e ansiedade. Esses achados justificam aqueles encontrados neste estudo, mas não foram verificadas diferenças nos níveis de ansiedade entre os diferentes grupos.

Em suma, os resultados ora apresentados evidenciam os benefícios que a participação em GTI proporciona para a função cognitiva e emocionalidade de idosos. A participação em GTI contempla a realização de atividades mistas e de frequência e intensidade variadas, que mesmo sendo simples, são de grande valia durante essa fase da vida. Já é sabido que durante a terceira idade, essa população tende a vivenciar uma segregação social, com diminuição de seus vínculos de amizades, caracterizando um quadro de exclusão social. ${ }^{33}$ Além disso, conforme exposto anteriormente, este fator, aliado a inatividade física e outras doenças e comorbidades, afeta a saúde cognitiva e física dos idosos, com grande repercussão na sua 
autonomia e qualidade de vida. Diante disso, torna-se necessário encontrar estratégias que viabilizem a atenção, promoção e prevenção da saúde, voltadas a esses segmentos populacionais.

Diante deste contexto, a participação em GTIs possibilita a convivência e a atenção coletiva. Além disso, esses grupos possibilitam o atendimento de grande número de idosos de forma conjunta e simultânea, atingindo vários idosos de determinada região, representando importante possibilidade de prática coletiva com resultados positivos para a saúde. Ainda em relação à participação de idosos em GTIs, Bulsing e colaboradores, ${ }^{34}$ ao avaliarem a influência dos grupos de convivência sobre a autoestima de idosas, verificaram que a participação em grupos de convivência é capaz de possibilitar o aumento do grupo de amizades, promovendo a ressocialização do idoso. Além disso, os GTIs também viabilizam a difusão de saberes, especialmente em assuntos referentes à saúde, temática de especial interesse para os idosos, devido ao empenho desses em ter um envelhecimento saudável. ${ }^{35}$

É importante salientar que este estudo apresentou limitações importantes, especialmente no que diz respeito ao recrutamento da amostra, que foi por conveniência. Embora esse tipo de amostra seja comumente utilizado, pode-se apenas sugerir que os achados encontrados se aplicam ao restante da população que apresenta as mesmas variáveis analisadas neste trabalho. Outro aspecto limitante relevante é o fato de

\section{REFERÊNCIAS}

1. Carvalho JAM, Garcia RA. O envelhecimento da população brasileira: um enfoque demográfico. Cad Saúde Pública 2003;19(3):725-33.

2. Veras R. Fórum. Envelhecimento populacional e as informações de saúde do PNAD: demandas e desafios contemporâneos. Introdução. Cad Saúde Pública 2007;23(10)2463-6. que, embora se tenha realizado avaliação geral do estado de saúde dos idosos, alguns idosos podem possuir outras patologias ainda não diagnosticadas que influenciariam os resultados do estudo.

Considerando o exposto, é possível constatar a necessidade de dar continuidade às investigações sobre esta temática. Pesquisas futuras podem ser realizadas, buscando analisar os efeitos da participação em GTIs na função cognitiva e estado emocional de idosos com uma metodologia longitudinal, de forma a analisar as estratégias utilizadas pelos GTIs e seu impacto funcional.

\section{CONCLUSÃO}

Neste estudo, foi verificado que a associação da diabetes mellitus com um estilo de vida mais inativo nos aspectos físicos, cognitivos e sociais, representa fator de risco para a aceleração das perdas cognitivas que acompanham o processo de envelhecimento, assim como estas variáveis também estão associadas com o desenvolvimento de depressão em indivíduos idosos.

Desta forma, o presente estudo corrobora a premissa de que a participação de idosos em grupos de terceira idade contribui positivamente para a manutenção de um envelhecimento saudável, uma vez que engloba momentos de lazer, práticas de exercício físico e atividades cognitivas, os quais são potentes fatores para a prevenção do declínio cognitivo e promoção da saúde do idoso.

3. Alencar N, De Souza JV Júnior, Aragão JCB, Ferreira MA, Dantas E. Nível de atividade física, autonomia funcional e qualidade de vida em idosas ativas e sedentárias. Fisioter mov 2010;23(3):473-81.

4. Burke SN, Barnes CA. Neural plasticity in the ageing brain. Nat Rev Neurosci 2006;7:30-40. 
5. Moscovitch M. Learning and memory. In: Baars BJ, Gage NM, editors. Cognition, brain, and consciousness. San Diego: Academic Press, 2007. p. 254-90.

6. Izquierdo I. Memória. Porto Alegre: Artmed; 2011.

7. Passos VM, Barreto SM, Diniz LM, Lima-Costa MF. Type 2 diabetes: prevalence and associated factors in a Brazilian community: the Bambuí health and aging study. São Paulo Med J 2005;123(2):66-71.

8. Sociedade Brasileira de Diabetes. Diretrizes da Sociedade Brasileira de Diabetes 2009. [3. ed.]. Itapevi, SP: A. Araújo Silva farmacêutica; 2009.

9. Wild S, Roglic G, Green A, Sicree R, King H. Global prevalence of diabetes:estimates for the year 2000 and projections for 2030. Diabetes Care 2004;27(5):1047-53.

10. National Institute of Diabetes and Digestive and Kidney Diseases, National Diabetes Information Clearinghouse. National Diabetes Statistics 2011. Bethesda: NDIC; 2011.

11. American Diabetes Association. Standards of Medical Care in diabetes 2010. Diabetes Care 2010;33(Suppl 1):S11-61.

12. Ozturk A, Simsek TT, Yümin ET, Sertel M, Yümin M. The relationship between physical, functional capacity and quality of life (QoL) among elderly people with a chronic disease. Arch Gerontol Geriatr 2001;53(3):278-83.

13. Lu FP, Lin KP, Kuo HK. Diabetes and the risk of multi-system aging phenotypes: a systematic review and meta-analysis. PLoS ONE 2009; 4(1):1-12.

14. Sims-Robinson, Kim B, Rosko A, Feldman EL. How does diabetes accelerate Alzheimer disease pathology? Nat Rev Neurol 2010;6(10):551-9.

15. Mello PB, Benetti F, Cammarota M, Izquierdo I. Physical exercise can reverse the deficit in fear memory induced by maternal deprivation. Neurobiol Learn Mem 2009;92(3):364-9.

16. Franco-Martin M, Parra-Vidales E, González-Palau F, Bernate-Navarro M, Solis A. [The influence of physical exercise in the prevention of cognitive deterioration in the elderly: a systematic review]. Rev Neurol 2013;56(11):545-54. Espanhol.

17. Balsamo S, Willardson J, De Santana FS, Prestes J, Balsamo DC, Nascimento DC, et al. Effectiveness of exercise on cognitive impairment and Alzheimer's disease. Intern J Gen Med 2013;6:387-91.

18. Souza JN, Chaves EC. O efeito do exercício de estimulação da memória em idosos saudáveis. Rev Esc Enferm USP 2005;39(1):13-9.
19. Oliveira CS, Costa SRR, Santos ICL, Lemos CES. Oficina de educação, memória, esquecimento e jogos lúdicos para a terceira idade. Rev Ciênc Ext 2012;8(1):8.

20. Folstein MF, Folstein SE, Mchugh PR. "Mini-Mental State": a practical method for grading the cognitive state of patients for the clinician. J Psychiatr Res 1975;12(3):189-98.

21. Almeida OP. Mini exame do estado mental e o diagnóstico de demência no Brasil. Arq Neuropsiquiatr 1998;56(3 B):605-12.

22. Andrade VM, Oliveira MG, Miranda MC, Oliveira AS, Oliveira EM, Bueno OF. Semantic relations and repetition of items enhance the free recall of words by multiple sclerosis patients. J Clin Exp Neuropsychol 2003;25(8):1070-78.

23. Martins IP, Loureiro C, Rodrigues S, Dias B. Nomeação de faces famosas: capacidade de evocação de nomes próprios numa amostra populacional portuguesa. Rev Psicol Educ Cult 2005;9(2):421-36.

24. Spreen O, Strauss E. A compendium of neuropsychological tests. 2. ed. New York: Oxford University Press; 1998.

25. Andreatini R, Seabra ML. A estabilidade do IDATEtraço: avaliação após cinco anos. Rev ABP-APAL 1993;15(1):21-5.

26. Coelho C, Burini R. Atividade física para prevenção e tratamento das doenças crônicas não transmissíveis e da incapacidade funcional. Rev Nutr 2009;22(6):937-46.

27. Friedland RP, Fritsch T, Smyth KA, Koss E, Lerner AJ, Chien HC, et al. Patients with Alzheimer's disease have reduced activities in midlife compared with healthy control-group members. Proc Nat Acad Sci USA 2001;98(6):3440-45.

28. Hall CB, Lipton RB, Sliwinski M, Katz J, Derby CA, Verghese J. Cognitive activities delay onset of memory decline in persons who develop dementia. Neurology 2009;73(5):356-61.

29. Sorman DE, Sorman DE, Sundstron A, Ronnlund M, Adolfsson R, Nilsson LG. Leisure activity in old age and risk of dementia: a 15-year prospective study. J Geront Ser B, Psychol Sci Soc Sci 2013:1-9.

30. Roy T, Lloyd CE. Epidemiology of depression and diabetes: a systematic review. J Affect Disord 2012;142 Suppl :S8-21.

31. Silva N, Atlantis E, Ismail K. A review of the association between depression and insulin resistance: pitfalls of secondary analyses or a promising new approach to prevention of type 2 diabetes? Curr Psychiatry Rep 2012;14(1):8-14. 
32. Campbell HM, Khan N, Cone C, Raisch DW.

Relationship between diet, exercise habits, and health status among patients with diabetes. Res Social Adm Pharm 2011;7(2):151-61.

33. Dalmolin IS, Leite MT, Hildebrandt LM, Sassi MM, Perdonssini LGB. A importância dos grupos de convivência como instrumento para a inserção social de idosos. Rev Contexto Saúde 2011;10(20):1-4.
34. Bulsing FL, de Oliveira KF, Da Rosa LMK, Da Fonseca L, Areosa SVC. A influência dos grupos de convivência sobre a auto-estima das mulheres idosas do município de Santa Cruz do Sul, RS. Rev Bras Ciênc Envelh Hum 2007;4(1):11-7.

35. Vargas LS, Alves N, Lara MVS, Mello-Carpes PB. Conscientizando idosos e profissionais da saúde acerca das mudanças cognitivas relacionadas à idade. Rev Ciênc Ext 2013;9(3):1-13.

Recebido: 18/9/2013

Revisado: $23 / 4 / 2014$

Aprovado: 22/7/2014 